\title{
UNITARY REPRESENTATIONS CORRESPONDING TO MEASURES WITH BOUNDED SUPPORT IN INFINITE DIMENSIONS
}

\author{
by JOSÉ E. GALÉ \\ (Received 28th July 1981)
}

\section{Introduction}

Let $E$ be a real Hausdorff locally convex space with topological dual $E^{\prime}$, topologised by the strong topology. Let $\left(x, x^{\prime}\right)$ denote the bilinear mapping defining the duality between $E$ and $E^{\prime}\left(x \in E, x^{\prime} \in E^{\prime}\right)$. By a unitary representation of $E^{\prime}$ we mean an operatorvalued function $U\left(x^{\prime}\right)=U_{x^{\prime}}$ defined on $E^{\prime}$, whose values are unitary operators in a separable Hilbert space $H$ such that

$$
U_{x_{1}^{\prime}+x_{2}^{\prime}}=U_{x_{1}^{\prime}} \circ U_{x_{2}^{\prime}}, \quad x_{1}^{\prime}, x_{2}^{\prime} \in E^{\prime}
$$

$U$ is called cyclic if there exists a vector $h \in H$ such that $\left\{U_{x^{\prime}} h: x^{\prime} \in E^{\prime}\right\}$ is total. Without loss of generality we may suppose that $\|h\|=(h, h)_{H}^{1 / 2}=1$ (by $\left(h_{1}, h_{2}\right)_{H}$ we denote the inner product on $\left.H, h_{1}, h_{2} \in H\right)$. The vector $h$ is called a cyclic vector for the representation $U$. Let $\mathscr{L}(H)$ denote the space of operators on $H$ with the norm topology. We call $U$ strongly continuous if the mapping $x^{\prime} \in E^{\prime} \mapsto U_{x^{\prime}} v \in H$ is continuous for each $v \in H$. Let $\mathbb{R}$ be the field of real numbers and let $n$ be a positive integer. Then, if $E^{\prime} \cong \mathbb{R}^{n}$, the following result is obtained from Bochner's theorem.

Theorem. Let $U: x^{\prime} \in \mathbb{R}^{n} \mapsto U_{x^{\prime}} \in \mathscr{L}(H)$ be a strongly continuous cyclic unitary representation with cyclic vector $h$. Then,

(i) There exists a Radon probability $\mu$ on $\mathbb{R}^{n}$ such that

$$
\left(U_{x}, h, h\right)_{H}=\int_{\mathbf{R}^{n}} \exp \left(i\left(x, x^{\prime}\right)\right) d \mu(x), \quad x^{\prime} \in \mathbb{R}^{n} .
$$

(ii) There exists an isometry between $H$ and $L^{2}(\mu)$ which transforms $U_{x^{\prime}}$ into the operator of multiplication by $\exp \left(i\left(x, x^{\prime}\right)\right), x^{\prime} \in E^{\prime}$.

It is natural to ask if this theorem is true in general for infinite dimensional $E$. The answer is positive if, for instance, $E$ is quasicomplete and $E^{\prime}$ is nuclear, as is well known (see [5], p. 365, Th. 5; [10], p. 236, Cor. 2 and p. 233, examples). When $E$ is a separable Hilbert space the theorem is not true, since the unitary representation that may be associated to the Gaussian probability is strongly continuous, for instance. There exists actually a bijection between the strongly continuous cyclic unitary representations and the cylindrical probabilities on $E$ which are scalarly concentrated on the balls of $E$ (see 
[10], p. 187, Prop. 2; p. 192, Def. 1; p. 193, Th. 1). In this paper we characterise the representations corresponding to Radon probabilities with bounded support. We use a theorem of Bochner type for the Fourier transforms of such measures. Finally, similar results are proved for semi-reflexive dual nuclear spaces.

By a Radon measure with bounded support on a locally convex space $E$ we mean a Radon measure on $E$, concentrated on some bounded subset of $E$ ([4], p. 116). Such measures on Hilbert spaces have been considered in [7] in relation to the Navier-Stokes equation. In [2], the authors study some special problems for measures with compact support on the real line.

\section{Hilbert space case}

In this section we suppose that $E$ is a real separable Hilbert space. Let $\mu$ be a Radon probability on $E$ for the weak topology, concentrated on a closed ball centered at origin $\Omega \subset E$. We consider the Hilbert space $L^{2}(\mu)$, topologised by the usual norm. If $x^{\prime} \in E^{\prime}$, the operator of multiplication by $\exp \left(i\left(., x^{\prime}\right)\right)$ is a unitary operator on $L^{2}(\mu)$ whose adjoint is the multiplication by $\exp \left(-i\left(., x^{\prime}\right)\right)$. We denote it by $M_{x^{\prime}}$. We consider the representation $x^{\prime} \in E^{\prime} \mapsto M_{x^{\prime}} \in \mathscr{L}\left(L^{2}(\mu)\right)$. If $x^{\prime}$ converges to $x_{0}^{\prime}$ in $E^{\prime}, \exp \left(i\left(., x^{\prime}\right)\right)$ converges to $\exp \left(i\left(., x_{0}^{\prime}\right)\right)$ uniformly on the ball $\Omega$; therefore this representation is continuous (not only strongly continuous!). Moreover, the set of linear combinations of the functions $\exp \left(i\left(., x^{\prime}\right)\right)$, $x \in E^{\prime}$, is dense for the topology of uniform convergence on $\Omega$ in the space of complex weakly continuous functions on $\Omega([4], \mathrm{p} .45 ; \mathrm{p}$. 105). In turn, the last space is dense in $L^{2}(\mu)$. It follows that the vector $f_{0} \equiv 1$ is cyclic for the representation $M$. Let $\mathbb{C}$ be the field of complex numbers. Let $E_{\mathrm{C}}^{\prime}$ denote the complexified space from $E^{\prime}$ with the product topology. We may extend that representation to $E_{\mathfrak{C}}^{\prime}$ by

$$
M_{z^{\prime}}(f)=\exp \left(i\left(., z^{\prime}\right)\right) f(.), \quad f \in L^{2}(\mu),
$$

where

$$
\left(x, z^{\prime}\right)=\left(x, x^{\prime}\right)+i\left(x, y^{\prime}\right), \quad x \in E,
$$

if $z^{\prime}=x^{\prime}+i y^{\prime} \in E_{\mathrm{C}}^{\prime}$

The fact that $\mu$ is concentrated on $\Omega$ implies $M_{z^{\prime}} \in \mathscr{L}\left(L^{2}(\mu)\right)$ (not unitary!). The mapping $z^{\prime} \in E_{, g}^{\prime} \mapsto M_{z^{\prime}} \in \mathscr{L}\left(L^{2}(\mu)\right)$ is continuous for the same reason as above. It is also a $G$-holomorphic function. Indeed, if $z_{1}^{\prime}, z_{2}^{\prime} \in E_{\mathbb{C}}^{\prime}$ and $g(\lambda)=M_{z_{1}^{\prime}+\lambda z_{2}^{\prime}}^{\prime}, \quad \lambda \in \mathbb{C}$, then $I=\int_{\gamma} g(\lambda) d \lambda \in \mathscr{L}\left(L^{2}(\mu)\right)$ for each closed path $\gamma \subset \mathbb{C}$, If $f_{1}, f_{2} \in L^{2}(\mu)$,

$$
\begin{aligned}
I\left(f_{1}\right)\left(f_{2}\right) & \left.=\int_{y} \int_{\Omega} \exp \left(i\left(x, z_{1}^{\prime}\right)\right) \exp \left(i\left(x, z_{2}^{\prime}\right)\right) \lambda\right) f_{1}(x) \overline{f_{2}(x)} d \mu(x) d \lambda \\
& =\int_{\Omega} \int_{\gamma} \exp \left(i\left(x, z_{1}^{\prime}\right)\right) f_{1}(x) \overline{f_{2}(x)} \exp \left(i\left(x, z_{2}^{\prime}\right) \lambda\right) d \lambda d \mu(x)=0,
\end{aligned}
$$

whence $g$ is an entire function (vectorial Morera's theorem). Finally, $\left\|M_{z^{\prime}}\right\|$ 
$=\sup \left\{\left\|M_{z^{\prime}}(f)\right\|_{2}:\|f\|_{2} \leqq 1\right\}=\exp \left(r\left\|\operatorname{Im} z^{\prime}\right\|\right)$, where $r$ is the radius of $\Omega, \operatorname{Im} z^{\prime}=y^{\prime}$, if $z^{\prime}=x^{\prime}$ $+i y^{\prime}, x^{\prime}, y^{\prime} \in E^{\prime}$. In short, we have verified that the representation $x^{\prime} \in E^{\prime} \mapsto M_{x^{\prime}} \in \mathscr{L}\left(L^{2}(\mu)\right)$ admits an entire extension to $E_{\mathrm{C}}^{\prime}$ such that $\left\|M_{z^{\prime}}\right\| \leqq \exp \left(r\left\|\operatorname{Im} z^{\prime}\right\|\right), z^{\prime} \in E_{\mathbb{C}}^{\prime}$, for certain $r \geqq 0$. Now, we proceed to prove the reciprocal assertion.

The following lemma is a consequence of Prokhorov's theorem and the arguments of [10], p. 189, Prop. 3, for cylindrical probabilities in Hilbert spaces. Let $\left\{P_{n}\right\}_{n=1}^{\infty}$ be the sequence of projections associated to a fixed orthonormal basis in $E$.

Lemma. Let $\left(\mu_{n}\right)_{n=1}^{\infty}=\left(\mu_{E / P_{n}(E) \perp}\right)_{n=1}^{\infty}$ be a cylindrical probability on $E$ such that there exists a ball $\Omega \subset E$, centered at origin, with $\mu_{n}\left(P_{n}(E) \backslash P_{n}(\Omega)\right)=0, n \in \mathbb{N}$. Then, there exists a $\sigma\left(E, E^{\prime}\right)$-Radon probability $\mu$ on $E$, concentrated on $\Omega$, with $\mu=\left(\mu_{n}\right)_{n=1}^{\infty}$.

Theorem 1.1. Let $\mu$ be a Radon probability on $E$, concentrated on the closed ball $\Omega_{r}$ $=\{x \in E:\|x\| \leqq r\}, r>0$. Let

$$
\hat{\mu}\left(x^{\prime}\right)=\int_{\Omega_{r}} \exp \left(i\left(x, x^{\prime}\right)\right) d \mu(x), \quad x^{\prime} \in E^{\prime},
$$

be the Fourier transform of $\mu$. Then,

(i) $\hat{\mu}$ is a continuous function of positive type on $E^{\prime}$ such that $\hat{\mu}(0)=1$.

(ii) $\hat{\mu}$ may be extended to an entire function $\theta$ on $E_{\mathrm{C}}^{\prime}$ such that $\left|\theta\left(z^{\prime}\right)\right| \leqq \exp \left(r \| \operatorname{Im} z^{\prime}||\right)$, $z^{\prime} \in E_{\mathrm{C}}^{\prime}$.

Conversely, if $\theta$ is an entire function satisfying (ii) and whose restriction to $E^{\prime}$ satisfies (i) there exists a Radon probability $\mu$ on E, concentrated on $\Omega_{r}$ and such that $\hat{\mu}=\theta$ on $E^{\prime}$.

Proof. Bochner's theorem for Hilbert spaces ([10], p. 239, Th. 3) proves part (i). Part (ii) is proved as for the representations. Conversely, part (i) implies that there is a cylindrical probability $\left(\mu_{n}\right)_{n=1}^{\infty}$ on $E$ such that if

$$
\theta_{n}: x_{n}^{\prime} \mapsto \theta\left(x_{n}^{\prime} \circ P_{n}\right), \quad x_{n}^{\prime} \in E_{n}^{\prime}=P_{n}(E)^{\prime}, \quad n \in \mathbb{N},
$$

then $\mu_{n}\left(x_{n}^{\prime}\right)=\theta_{n}\left(x_{n}^{\prime}\right)$.

([10], p. 187, Prop. 2). Moreover the function

$$
z_{n}^{\prime} \in E_{n_{\mathcal{C}}}^{\prime} \mapsto \theta_{n}\left(z_{n}^{\prime}\right)=\theta\left(z_{n}^{\prime} \circ P_{n}\right)
$$

is entire for each $n$ and it verifies

$$
\left|\theta_{n}\left(z_{n}^{\prime}\right)\right| \leqq \exp \left(r\left\|\operatorname{Im} z_{n}^{\prime}\right\|\right) \quad \text { for every } \quad z_{n}^{\prime} \in E_{n_{C}}^{\prime}
$$

(we still denote by $P_{n}$ the obvious extension of $P_{n}$ on $E_{\mathbb{C}}$ ). Because of the Paley-Wiener theorem there is a distribution $T_{n}$ with support contained in the ball of radius $r$ in $E_{n}$ $=P_{n}(E) \cong \mathbb{R}^{n}$ such that

$$
\widehat{T}_{n}\left(x_{n}^{\prime}\right)=T_{n}\left(\exp \left(i\left(., x_{n}^{\prime}\right)\right)\right)=\theta_{n}\left(x_{n}^{\prime}\right)=\hat{\mu}_{n}\left(x_{n}^{\prime}\right), \quad x_{n}^{\prime} \in \mathbb{R}^{n} .
$$


Let $\mathscr{S}_{n}$ be the space of all functions rapidly decreasing at infinity in $\mathbb{R}^{n}$. Let $\alpha, \beta \in \mathscr{S}_{n}$ so that $\beta$ is the Fourier transform of $\alpha, \beta=\hat{\alpha}$. Then,

$$
\begin{aligned}
T_{n}(\beta)=T_{n}(\hat{\alpha}) & =\int_{\mathbb{R}^{n}} \hat{T}_{n}\left(x^{\prime}\right) \alpha\left(x^{\prime}\right) d x \\
& =\int_{\mathbb{R}^{n}} \hat{n}_{n}\left(x^{\prime}\right) \alpha\left(x^{\prime}\right) d x^{\prime} \\
& =\int_{\mathbb{R}^{n}} \int_{\mathbb{R}^{n}} \alpha\left(x^{\prime}\right) \exp \left(i\left(x, x^{\prime}\right)\right) d \mu_{n}(x) d x^{\prime} \\
& =\int_{\mathbb{R}^{n}} \hat{\alpha}(x) d \mu_{n}(x)=\int_{\mathbb{R}^{n}} \beta(x) d \mu_{n}(x) .
\end{aligned}
$$

Therefore, the distributions $\mu_{n}, T_{n}$ coincide on $\mathscr{S}_{n}$ and thus $\mu_{n}=T_{n}(n \in \mathbb{N})$. It follows that $\mu_{n}$ has support contained in $\Omega_{r}$ and, according to the preceding lemma, there exists a Radon probability $\mu$ on $E$, concentrated on $\Omega_{r}$, such that $\mu=\left(\mu_{n}\right)_{n=1}^{\infty}$.

Theorem 1.2. Let $U: x^{\prime} \in E^{\prime} \mapsto U_{x^{\prime}} \in \mathscr{L}(H)$ be a continuous cyclic unitary representation with cyclic vector $h$. If $U$ admits an entire extension $\tilde{U}: E_{\mathrm{c}}^{\prime} \mapsto \mathscr{L}(H)$ verifying $\left\|\widetilde{U}_{z^{\prime}}\right\| \leqq \exp \left(r\left\|\operatorname{Im} z^{\prime}\right\|\right), z^{\prime} \in E_{\mathrm{C}}^{\prime}$, for a certain $r>0$, then

(i) There exists a Radon probability $\mu$ on $E$, concentrated on $\Omega_{r}$, such that

$$
\left(U_{x^{\prime}} h, h\right)_{H}=\int_{\Omega_{r}} \exp \left(i\left(x, x^{\prime}\right)\right) d \mu(x), \quad x^{\prime} \in E^{\prime} .
$$

(ii) The equality of part (i) defines an isometric correspondence between $H$ and $L^{2}(\mu)$, so that the operator corresponding by this isometry to $U_{x^{\prime}}$ is the operator of multiplication by $\exp \left(i\left(., x^{\prime}\right)\right)$.

Proof. It is standard. The function $x^{\prime} \in E^{\prime} \mapsto \theta\left(x^{\prime}\right)=\left(U_{x^{\prime}} h, h\right)_{H}$ is continuous and of positive type such that $\theta(0)=1$. Moreover $z^{\prime} \in E_{\mathrm{C}}^{\prime} \mapsto\left(\widetilde{U}_{z}, h, h\right)_{H}$ is an entire function and $\left|\theta\left(z^{\prime}\right)\right| \leqq\left\|\widetilde{U}_{z^{\prime}}\right\| \leqq \exp \left(r|| \operatorname{Im} z^{\prime}||\right), z^{\prime} \in E_{\mathrm{C}}^{\prime}$. Theorem (1.1) implies part (i). The isometry of part (ii) is proved by associating to each vector $\sum_{k=1}^{n} \lambda_{k} U_{x_{k}} h \in H$ the function $\sum_{k=1}^{n} \lambda_{k} \exp \left(i\left(., x_{k}^{\prime}\right)\right) \in L^{2}(\mu)$, and applying obvious arguments about density in $H$ and $L^{2}(\mu)$.

\section{Nuclear space case}

Henceforth we suppose that $E$ is a semi-reflexive (i.e. $E=E^{\prime \prime}$ algebraically) dual nuclear locally convex space (see [9]). Let $\mathscr{B}$ be the family of all subsets of $E$ which are closed, bounded, balanced and convex. If $B \in \mathscr{B}$, let $B^{0}$ denote the polar set of $B$ in $E^{\prime}$. We suppose $E^{\prime}$ endowed its strong topology or topology of uniform convergence on the elements of $\mathscr{B}$. This is defined by the seminorms $q_{B}\left(x^{\prime}\right)=\sup _{x \in B}\left|\left(x, x^{\prime}\right)\right|=\left\|x^{\prime}\right\|_{B}, x^{\prime} \in E^{\prime}$, where $q_{B^{0}}$ is the gauge of $B^{0} \subset E^{\prime}, B \in \mathscr{B}$. Let $E_{B}$ denote the linear subspace of $E$ spanned by $B$ and normed by the gauge of $B$. Let $E_{B^{0}}^{\prime}$ denote the quotient space $E^{\prime} / q_{B^{0}}^{-1}(0)$ topologised by the norm defined by $q_{B^{0}}$. If $x^{\prime} \in E^{\prime}$, let $\left[x^{\prime}\right]$ denote the image through the 
canonical surjection $E^{\prime} \rightarrow E_{B^{0}}^{\prime}$. Obviously, this surjection induces a surjection $\pi_{B}: E_{\mathrm{C}}^{\prime} \rightarrow\left(E_{B}^{\prime}\right)_{\mathrm{C}}$. If $\theta: E_{\mathrm{C}}^{\prime} \rightarrow \mathbb{C}$ is an entire function ( $G$-entire and continuous) we say that $\theta$ is factorisable if there is $B \in \mathscr{B}$ and $\eta:\left(E_{B 0}^{\prime}\right)_{\mathbb{C}} \rightarrow \mathbb{C}$, where $\eta$ is entire, such that $\theta=\eta \circ \pi_{B}$. By Radon probabilities we mean the ones relative to weak topology $\sigma\left(E, E^{\prime}\right)$.

Theorem 2.1. Let $\mu$ be a Radon probability on $E$, concentrated on $B \in \mathscr{B}$. Let $\hat{\mu}\left(x^{\prime}\right)$ $=\int_{B} \exp \left(i\left(x, x^{\prime}\right)\right) d \mu(x), x^{\prime} \in E^{\prime}$, be its Fourier transform. Then,

(i) $\hat{\mu}$ is a continuous function of positive type on $E^{\prime}$ and $\hat{\mu}(0)=1$.

(ii) $\mu$ may be extended to a factorisable entire function $\theta$ on $E_{\mathbb{C}}^{\prime}$ such that $\left|\theta\left(z^{\prime}\right)\right| \leqq \exp \left(\left\|\operatorname{Im} z^{\prime}\right\|_{B}\right), z^{\prime} \in E_{\mathrm{C}}^{\prime}$.

Conversely, if $\theta$ is an entire function on $E_{\mathscr{C}}^{\prime}$ satisfying (ii), whose restriction to $E^{\prime}$ satisfies (i) there exists a Radon probability $\mu$ on $E$, concentrated on some element of $\mathscr{B}$ and such that $\hat{\mu}=\theta$ on $E^{\prime}$.

Proof. Let $\mu$ be a Radon probability. Part (i) is a consequence of [10], p. 193, Th. 1 . It makes sense to define $\hat{\mu}\left(z^{\prime}\right)=\int_{B} \exp \left(i\left(x, z^{\prime}\right)\right) d \mu(x)$, for every $z^{\prime} \in E_{\mathfrak{C}}^{\prime}$, and it is easy to prove that $\hat{\mu}$ is a $G$-holomorphic and continuous function on $E_{\mathbb{C}}^{\prime}$.

Now, without loss of generality, we may suppose that $E_{B^{0}}^{\prime}$ is a separable pre-Hilbert space since $E^{\prime}$ is nuclear. The topological dual of $E_{B}^{\prime}{ }^{0}$ coincides with $E_{B 00}^{\prime \prime}=E_{B}$ ( $E$ is semireflexive) and the strong topology on $E_{B}$ is the one defined by the gauge of $B$. Thus $E_{B}$ is a separable Hilbert space whose topological dual is the completion of $E_{B^{0}}^{\prime}$. The continuity of the injection $E_{B} \rightarrow E$ implies the identity of topologies $\sigma\left(E_{B},\left(E_{B}\right)^{\prime}\right), \sigma\left(E, E^{\prime}\right)$ on $B$. Let $v$ be the measure $\mu$ considered as Radon measure on the Hilbert space $E_{B}$. According to previous arguments, the function $\hat{v}:\left(E_{B}^{\prime}\right)_{\mathbb{C}} \rightarrow \mathbb{C}$ in entire and, for $\left[x^{\prime}\right]$, $\left[y^{\prime}\right] \in E_{B^{0}}^{\prime}$,

$$
\begin{aligned}
\hat{v}\left(\left[x^{\prime}\right]+i\left[y^{\prime}\right]\right) & =\int_{B} \exp \left(i\left(x,\left[x^{\prime}\right]+i\left[y^{\prime}\right]\right) d \mu(x)\right. \\
& =\int_{B} \exp \left(i\left(x,\left[x^{\prime}\right]\right)\right) \exp \left(-\left(x,\left[y^{\prime}\right]\right)\right) d \mu(x) \\
& =\int_{B} \exp \left(i\left(x, x^{\prime}\right)\right) \exp \left(-\left(x, y^{\prime}\right)\right) d \mu(x)=\hat{\mu}\left(x^{\prime}+i y^{\prime}\right),
\end{aligned}
$$

i.e., $\hat{v} \circ \pi_{B}=\hat{\mu}$.

Conversely, we may suppose that $E_{B}$ is a Hilbert space and $\theta$ is factorisable through $E_{B 0}^{\prime}$, i.e., there is $\eta:\left(E_{B 0}^{\prime}\right)_{\mathbb{C}} \rightarrow \mathbb{C}$ an entire function, such that $\theta=\eta \circ \pi_{B}$. Evidently, the restriction of $\eta$ to $E_{B}^{\prime}$ is a continuous function of positive type. Moreover, for every $z^{\prime} \in E_{\complement}^{\prime}$,

$$
\left|\eta\left(\pi_{B} z^{\prime}\right)\right|=\left|\theta\left(z^{\prime}\right)\right|=\exp \left(\left\|\operatorname{Im} z^{\prime}\right\|_{B}\right)=\exp \left\|\operatorname{Im} \pi_{B} z^{\prime}\right\|_{B}
$$

Therefore, the natural extension $\bar{\eta}$ of $\eta$ to the completion of $\left(E_{B 0}^{\prime}\right)_{C}$ verifies (i) and (ii) of Theorem 1.1. Thus, there exists a Radon probability $\mu$ on $E_{B}$ concentrated on $B$, such that $\hat{\mu}\left(\left[x^{\prime}\right]\right)=\eta\left(\left[x^{\prime}\right]\right)=\theta\left(x^{\prime}\right)$ for every $x^{\prime} \in E^{\prime}$. If we consider $\mu$ as a Radon measure on $E$, then $\hat{\mu}\left(x^{\prime}\right)=\hat{\mu}\left(\left[x^{\prime}\right]\right)=\theta\left(x^{\prime}\right)$, for every $x^{\prime} \in E^{\prime}$, and the proof is finished. 
Remark. Theorem 2.1 is also true if the word "factorisable" is dropped in condition (ii), because every entire function $\theta$ on $E_{\mathrm{C}}^{\prime}$ satisfying

$$
\left|\theta\left(z^{\prime}\right)\right| \leqq \exp \left(\left\|\operatorname{Im} z^{\prime}\right\|_{B}\right), \quad z^{\prime} \in E_{\mathfrak{C}}^{\prime}
$$

is factorisable. It is enough to prove that $\theta\left(z_{1}^{\prime}\right)=\theta\left(z_{2}^{\prime}\right)$ for $z_{1}^{\prime}, z_{2}^{\prime} \in E_{\mathbb{C}}^{\prime}$ with $z_{1}^{\prime}=z_{2}^{\prime}$ on $B$. Using Cauchy's inequalities the last identity is verified by each $n$-linear mapping of the Taylor series of $\theta$ at the origin. Therefore, it is also verified by $\theta$.

By means of arguments similar to those of Sections 1 and 2 and some simple additions, we obtain a bijective correspondence similar to that of Theorem 1.2.

Remark. We may state analogous results in the setting of bornological linear spaces, under suitable restrictions.

Acknowledgments. I am grateful to Prof. M. Paramio and J. L. Rubio de Francia for helpful talks about this paper.

\section{REFERENCES}

1. T. Abuabara, A version of the Paley-Wiener-Schwartz theorem in Infinite Dimensions, Advances in Holomorphy, J. A. Barroso (ed.), Proc. of Seminário de Holomorfia (Rio de Janeiro 1977) (North-Holland, Amsterdam-New York-Oxford, 1979), 1-29.

2. A. Beurling and P. Malliavin, On Fourier transforms of measures with compact support, Acta Math. 107 (1962), 291-309.

3. J. F. Colombeau and J. M. Ansémil, The Paley-Wiener-Schwartz isomorphism in Nuclear spaces, Rèvue Roumaine de Math. Pures et Appliquées (to appear).

4. J. E. GaLÉ, Sobre espacios de funciones diferenciables en dimensión infinita, aproximables por polinomios sobre conjuntos acotados (Tesis Doctoral, Facultad de Ciencias, Zaragoza, 1980).

5. I. M. Gelfand and N. Ya. Vilenkin, Generalized Function, vol. 4. Applications of Harmonic Analysis (Academic Press, New York-London, 1964).

6. H. Hogbe-Nlend, Théorie des Bornologies et Applications (Lecture Notes in Math. no. 213, Berlin-Heidelberg-New York, 1971).

7. O. A. Ladyzhenskaya and A. M. Vershik, Sur l'évolution des mesures détérminées par les équations de Navier-Stokes et la résolution du problème de Cauchy pour l'équation statistique de E. Hopf, Annali della Scuola Norm. Sup. di Pisa. Serie IV, 4 (1977), 209-230.

8. L. NAChis and S. Dineen, Entire functions of exponential type bounded on the real axis and Fourier transforms of distributions with bounded supports, Israel J. Math. 13 (1976), 321326.

9. A. Pietsch, Nuclear Locally Convex Spaces (Ergebnisse der Mathematik und ihrer Grenzgebiete no. 66 (Second Edition), Springer, Berlin-Heidelberg-New York, 1972).

10. L. Schwarz, Radon Measures on Arbitrary Topological Spaces and Cylindrical Measures, Tata Institute of Fundamental Research (Studies in Math. no. 6, Oxford University Press, 1973).

Departamento de Teoria de Funciones

Facultad de Ciencias, Zaragoza

Spain 